\title{
Existence of Positive Solutions for Higher Order $(p, q)$-Laplacian Two-Point Boundary Value Problems
}

\author{
Rajendra Prasad Kapula, ${ }^{1}$ Penugurthi Murali, ${ }^{1}$ and Kona Rajendrakumar ${ }^{2}$ \\ ${ }^{1}$ Department of Applied Mathematics, Andhra University, Visakhapatnam 530003, India \\ ${ }^{2}$ Department of Mathematics, VITAM College of Engineering, Visakhapatnam 531173, India
}

Correspondence should be addressed to Rajendra Prasad Kapula; rajendra92@rediffmail.com

Received 17 April 2013; Revised 17 July 2013; Accepted 17 July 2013

Academic Editor: Kanishka Perera

Copyright (c) 2013 Rajendra Prasad Kapula et al. This is an open access article distributed under the Creative Commons Attribution License, which permits unrestricted use, distribution, and reproduction in any medium, provided the original work is properly cited.

We derive sufficient conditions for the existence of positive solutions to higher order $(p, q)$-Laplacian two-point boundary value problem, $(-1)^{m_{1}+n_{1}-1}\left[\phi_{p}\left(u^{\left(2 m_{1}\right)}(t)\right)\right]^{\left(n_{1}\right)}=f_{1}(t, u(t), v(t)), t \in[0,1],(-1)^{m_{2}+n_{2}-1}\left[\phi_{q}\left(v^{\left(m_{2}\right)}(t)\right)\right]^{\left(2 n_{2}\right)}=f_{2}(t, u(t), v(t)), t \in$ $[0,1], u^{(2 i)}(0)=0=u^{(2 i)}(1), i=0,1,2, \ldots, m_{1}-1,\left[\phi_{p}\left(u^{\left(2 m_{1}\right)}(t)\right)\right]_{\text {at } t=0}^{(j)}=0, j=0,1, \ldots, n_{1}-2 ;\left[\phi_{p}\left(u^{\left(2 m_{1}\right)}(1)\right)\right]=0,\left[\phi_{q}\left(v^{\left(m_{2}\right)}(t)\right)\right]_{\text {at } t=0}^{(2 i)}=$ $0=\left[\phi_{q}\left(v^{\left(m_{2}\right)}(t)\right)\right]_{\text {at } t=1}^{(2 i)}, i=0,1, \ldots, n_{2}-1, v^{(j)}(0)=0, j=0,1,2, \ldots, m_{2}-2$, and $v(1)=0$, where $f_{1}, f_{2}$ are continuous functions from $[0,1] \times \mathbb{R}^{2}$ to $[0, \infty), m_{1}, n_{1}, m_{2}, n_{2} \in \mathbb{N}$ and $1 / p+1 / q=1$. We establish the existence of at least three positive solutions for the two-point coupled system by utilizing five-functional fixed point theorem. And also, we demonstrate our result with an example.

\section{Introduction}

The goal of differential equations is to understand the physical phenomena of nature by developing mathematical models. Among all, a class of differential equations governed by nonlinear differential operators, which have wide applications and interest, has been developed to study such type of equations. In this theory, the most investigated operator is the classical $p$-Laplacian, given by $\phi_{p}(y):=y|y|^{p-2}$ with $p>1$. These problems have a wide range of applications in physics and related sciences such as biophysics, plasma physics, and chemical reaction design. Due to the importance in both theory and applications, $p$-Laplacian boundary value problems have created a great deal of interest in recent years; we mention a few [1-11].

Recently, Prasad and Murali [12] established the existence of positive solutions of $p$-Laplacian singular boundary value problem on time scale,

$$
\begin{array}{r}
\left(\phi_{p}\left(y^{\Delta}(t)\right)\right)^{\nabla}+q(t) f\left(t, y(t), y^{\Delta}(t)\right)=0, \\
t \in(0,1)_{\mathbb{T}},
\end{array}
$$

$$
y(0)=0=y^{\Delta}(1)
$$

by assuming suitable conditions on $f$. Till now in the literature of boundary value problems, the theory was not developed to the system of higher order boundary value problems with $(p, q)$-Laplacian. Mainly, this type of problems arises in radar invention models and microatom invention models.

Due to our interest in the literature, in this paper, we consider two-point higher order $(p, q)$-Laplacian boundary value problem (BVP)

$$
\begin{array}{r}
(-1)^{m_{1}+n_{1}-1}\left[\phi_{p}\left(u^{\left(2 m_{1}\right)}(t)\right)\right]^{\left(n_{1}\right)}=f_{1}(t, u(t), v(t)), \\
t \in[0,1], \\
(-1)^{m_{2}+n_{2}-1}\left[\phi_{q}\left(v^{\left(m_{2}\right)}(t)\right)\right]^{\left(2 n_{2}\right)}=f_{2}(t, u(t), v(t)), \\
t \in[0,1],
\end{array}
$$




$$
\begin{gathered}
u^{(2 i)}(0)=0=u^{(2 i)}(1), \quad i=0,1,2, \ldots, m_{1}-1, \\
{\left[\phi_{p}\left(u^{\left(2 m_{1}\right)}(t)\right)\right]_{\mathrm{at} t=0}^{(j)}=0, \quad j=0,1,2, \ldots, n_{1}-2,} \\
{\left[\phi_{p}\left(u^{\left(2 m_{1}\right)}(t)\right)\right]_{\mathrm{at} t=1}=0,} \\
{\left[\phi_{q}\left(v^{\left(m_{2}\right)}(t)\right)\right]_{\mathrm{at} t=0}^{(2 i)}=0=\left[\phi_{q}\left(v^{\left(m_{2}\right)}(t)\right)\right]_{\mathrm{at} t=1}^{(2 i)},} \\
v^{(j)}(0)=0, \quad j=0,1,2, \ldots, m_{2}-1, \quad v(1)=0,
\end{gathered}
$$

where $f_{1}, f_{2}$ are continuous functions from $[0,1] \times \mathbb{R}^{2}$ to $[0, \infty), m_{1}, n_{1}, m_{2}, n_{2} \in \mathbb{N}$ and $1 / p+1 / q=1$. If we take $p=q$ in the above problem then it reduces to $p$-Laplacian problem.

To obtain a solution of the BVP (2), we construct Green's functions for the corresponding homogeneous BVPs. For $n_{1} \geq 2$, let $G(t, s)$ be Green's function of the BVP,

$$
\begin{gathered}
(-1)^{n_{1}-1} x^{\left(n_{1}\right)}(t)=0, \quad t \in[0,1], \\
x^{(j)}(0)=0, \quad j=0,1,2, \ldots, n_{1}-2, \quad x(1)=0
\end{gathered}
$$

and it is given by

$$
G(t, s)= \begin{cases}\frac{t^{n_{1}-1}(1-s)^{n_{1}-1}}{\left(n_{1}-1\right) !}, & 0 \leq t \leq s<1, \\ \frac{t^{n_{1}-1}(1-s)^{n_{1}-1}}{\left(n_{1}-1\right) !}-\frac{(t-s)^{n_{1}-1}}{\left(n_{1}-1\right) !}, & 0<s \leq t \leq 1 .\end{cases}
$$

Let $H_{m_{1}}(t, s)$ be Green's function of the BVP

$$
\begin{gathered}
(-1)^{m_{1}} y^{\left(2 m_{1}\right)}(t)=0, \quad t \in[0,1], \\
y^{(2 i)}(0)=0=y^{(2 i)}(1), \quad i=0,1, \ldots, m_{1}-1,
\end{gathered}
$$

and it can be recursively defined as

$$
H_{m_{1}}(t, s)=\int_{0}^{1} H_{m_{1}-1}(t, r) H_{1}(r, s) d r
$$

where $H_{1}(t, s)$ is Green's function of

$$
-y^{\prime \prime}(t)=0, \quad y(0)=0=y(1)
$$

and is given by

$$
H_{1}(t, s)= \begin{cases}t(1-s), & 0 \leq t \leq s<1 \\ s(1-t), & 0<s \leq t \leq 1\end{cases}
$$

Then $u(t)$ can be expressed in the form

$$
\begin{aligned}
u(t)=\int_{0}^{1} H_{m_{1}}(t, s) \phi_{p}^{-1} \\
\quad \times\left(\int_{0}^{1} G(s, \tau) f_{1}(\tau, u(\tau), v(\tau)) d \tau\right) d s .
\end{aligned}
$$

Since $\phi_{p}^{-1}=\phi_{q}$, we have

$$
u(t)=\int_{0}^{1} H_{m_{1}}(t, s) \phi_{q}\left(\int_{0}^{1} G(s, \tau) f_{1}(\tau, u(\tau), v(\tau)) d \tau\right) d s
$$

For $n_{1}=1$,

$$
u(t)=\int_{0}^{1} H_{m_{1}}(t, s) \phi_{q}\left(\int_{s}^{1} f_{1}(\tau, u(\tau), v(\tau)) d \tau\right) d s .
$$

For $m_{2} \geq 2$, let $H(t, s)$ be Green's function of the BVP

$$
\begin{gathered}
(-1)^{m_{2}-1} x^{\left(m_{2}\right)}(t)=0, \quad t \in[0,1], \\
x^{(j)}(0)=0, \quad j=0,1,2, \ldots, m_{2}-2, \quad x(1)=0
\end{gathered}
$$

and it is given by

$$
H(t, s)= \begin{cases}\frac{t^{m_{2}-1}(1-s)^{m_{2}-1}}{\left(m_{2}-1\right) !}, & 0 \leq t \leq s<1, \\ \frac{t^{m_{2}-1}(1-s)^{m_{2}-1}}{\left(m_{2}-1\right) !}-\frac{(t-s)^{m_{2}-1}}{\left(m_{2}-1\right) !}, & 0<s \leq t \leq 1 .\end{cases}
$$

Let $G_{n_{2}}(t, s)$ be Green's function of the BVP

$$
\begin{gathered}
(-1)^{n_{2}} y^{\left(2 n_{2}\right)}(t)=0, \quad t \in[0,1], \\
y^{(2 i)}(0)=0=y^{(2 i)}(1), \quad i=0,1, \ldots, n_{2}-1,
\end{gathered}
$$

and it can be recursively defined as

$$
G_{n_{2}}(t, s)=\int_{0}^{1} G_{n_{2}-1}(t, r) G_{1}(r, s) d r,
$$

where $G_{1}(t, s)$ is Green's function of

$$
-y^{\prime \prime}(t)=0, \quad y(0)=0=y(1)
$$

and is given by

$$
G_{1}(t, s)= \begin{cases}t(1-s), & 0 \leq t \leq s<1 \\ s(1-t), & 0<s \leq t \leq 1\end{cases}
$$

Then $v(t)$ can be expressed in the form

$$
\begin{aligned}
v(t)=\int_{0}^{1} H(t, s) \phi_{q}^{-1} \\
\quad \times\left(\int_{0}^{1} G_{n_{2}}(s, \tau) f_{2}(\tau, u(\tau), v(\tau)) d \tau\right) d s .
\end{aligned}
$$

Since $\phi_{q}^{-1}=\phi_{p}$, we have

$$
v(t)=\int_{0}^{1} H(t, s) \phi_{p}\left(\int_{0}^{1} G_{n_{2}}(s, \tau) f_{2}(\tau, u(\tau), v(\tau)) d \tau\right) d s .
$$


For $m_{2}=1$,

$$
v(t)=\int_{t}^{1} \phi_{p}\left(\int_{0}^{1} G_{n_{2}}(s, \tau) f_{2}(\tau, u(\tau), v(\tau)) d \tau\right) d s .
$$

Further, it is easily seen that $H_{m_{1}}(t, s), H(t, s), G_{n_{2}}(t, s)$ and $G(t, s)$, all are nonnegative on $[0,1] \times[0,1]$.

A solution of the BVP (2) is a function $(u, v) \in C^{2 m_{1}}$ $[0,1] \times C^{m_{2}}[0,1]$ such that $\left(\phi_{p} \circ u^{\left(2 m_{1}\right)}, \phi_{q} \circ v^{\left(m_{2}\right)}\right) \in C^{n_{1}}[0,1] \times$ $C^{2 n_{2}}[0,1]$, and $(u, v)$ satisfies the BVP $(2)$.

A positive solution $(u, v)$ of the BVP $(2)$ is a solution of the BVP (2) such that $u$ and $v$ are nonnegative on $[0,1]$.

The rest of the paper is organized as follows. In Section 2, we estimate the bounds of Green's functions. In Section 3, we establish the existence of at least three positive solutions for two-point BVP (2) by using Avery's generalization of LeggettWilliams fixed point theorem. And also, we demonstrate our result with an example.

\section{Bounds of Green's Functions}

In this section, we state some lemmas to estimate bounds on Green's functions which are needed in later discussions. The following lemma is included in the paper to prove the remaining lemmas.

Lemma 1. Let $w \in(0,(b-a) / 2]$ and $I=[a+w, b-w]$. Then, for any $j \in \mathbb{N}$

$$
G_{j}(t, s) \geq\left(\frac{w}{b-a}\right)^{j}\left(\phi_{w}\right)^{j-1} G_{1}(s, s), \quad \forall(t, s) \in I \times[a, b],
$$

where $\phi_{w}=\int_{a+w}^{b-w} G_{1}(r, r) \nabla r>0$.

Proof. The proof is by induction. First, for $j=1$ inequality (21) is obvious. Next, for fixed $j$, assume that (21) is true; from recursive formula we have, for each $(t, s) \in I \times[a, b]$,

$$
\begin{aligned}
G_{j+1} & (t, s) \\
& =\int_{a}^{b} G_{j}(t, r) G_{1}(r, s) \nabla r \\
& \geq \int_{a}^{b}\left(\frac{w}{b-a}\right)^{j}\left(\phi_{w}\right)^{j-1} G_{1}(r, r) \cdot G_{1}(r, s) \nabla r \\
& \geq\left(\frac{w}{b-a}\right)^{j}\left(\phi_{w}\right)^{j-1} \int_{a+w}^{b-w} G_{1}(r, r) \cdot G_{1}(r, s) \nabla r \\
& \geq\left(\frac{w}{b-a}\right)^{j}\left(\phi_{w}\right)^{j-1} \int_{a+w}^{b-w} G_{1}(r, r) \cdot\left(\frac{w}{b-a}\right) G_{1}(s, s) \nabla r \\
& =\left(\frac{w}{b-a}\right)^{j+1}\left(\phi_{w}\right)^{j} G_{1}(s, s) .
\end{aligned}
$$

Hence, by induction the proof is complete.

In the previous lemma by choosing $a=0, b=1$, and $w=1 / 4, w=1 / 3$, and $w=1 / 8$, we get $I=[1 / 4,3 / 4]$,
$I_{1}=[1 / 3,2 / 3]$, and $I_{2}=[1 / 8,7 / 8]$. We know that $G_{1}(s, s)=$ $s(1-s)$.

Lemma 2. For $(t, s) \in I \times[0,1]$, one has

$$
G_{j}(t, s) \geq\left(\frac{11}{6}\right)^{j-1} \frac{1}{4^{3 j-2}}(1-s) s .
$$

For $(t, s) \in I_{1} \times[0,1]$, one has

$$
G_{j}(t, s) \geq \frac{1}{3}\left(\frac{13}{486}\right)^{j-1}(1-s) s .
$$

For $(t, s) \in I_{2} \times[0,1]$, one has

$$
G_{j}(t, s) \geq \frac{1}{8}\left(\frac{39}{2048}\right)^{j-1}(1-s) s,
$$

where $j=1,2, \ldots, n_{2}$.

Lemma 3. For $t, s \in[0,1]$, one has

$$
G_{j}(t, s) \leq \frac{1}{6^{j-1}}(1-s) s,
$$

where $j=1,2, \ldots, n_{2}$.

For details refer to [9].

In Lemma 1 , replacing $G_{j}(\cdot, \cdot)$ by $H_{j}(\cdot, \cdot)$, we have the following.

Lemma 4. For $(t, s) \in I \times[0,1]$, one has

$$
H_{j}(t, s) \geq\left(\frac{11}{6}\right)^{j-1} \frac{1}{4^{3 j-2}}(1-s) s .
$$

For $(t, s) \in I_{1} \times[0,1]$, one has

$$
H_{j}(t, s) \geq \frac{1}{3}\left(\frac{13}{486}\right)^{j-1}(1-s) s .
$$

For $(t, s) \in I_{2} \times[0,1]$, one has

$$
H_{j}(t, s) \geq \frac{1}{8}\left(\frac{39}{2048}\right)^{j-1}(1-s) s,
$$

where $j=1,2, \ldots, m_{1}$.

Lemma 5. For $t, s \in[0,1]$, one has

$$
H_{j}(t, s) \leq \frac{1}{6^{j-1}}(1-s) s,
$$

where $j=1,2, \ldots, m_{1}$.

For details refer to [9].

Lemma 6. For $(t, s) \in I \times[0,1]$, one has

$$
H(t, s) \geq \frac{1}{4^{m-1}} H(s, s) .
$$

For $(t, s) \in I_{1} \times[0,1]$, one has

$$
H(t, s) \geq \frac{1}{3^{m-1}} H(s, s) .
$$

For $(t, s) \in I_{2} \times[0,1]$, one has

$$
H(t, s) \geq \frac{1}{8^{m-1}} H(s, s) .
$$

For details refer to [11]. 
Lemma 7. For $t, s \in[0,1]$, one has

$$
H(t, s) \leq H(s, s) .
$$

For details refer to [11].

Lemma 8. For $(t, s) \in I \times[0,1]$, one has

$$
G(t, s) \geq \frac{1}{4^{n-1}} G(s, s) .
$$

For $(t, s) \in I_{1} \times[0,1]$, one has

$$
G(t, s) \geq \frac{1}{3^{n-1}} G(s, s) .
$$

For $(t, s) \in I_{2} \times[0,1]$, one has

$$
G(t, s) \geq \frac{1}{8^{n-1}} G(s, s) .
$$

For details refer to [11].

Lemma 9. For $t, s \in[0,1]$, one has

$$
G(t, s) \leq G(s, s) \text {. }
$$

For details refer to [11].

Denote

$$
\begin{array}{r}
\mathscr{M}=\min \left\{\left(\frac{11}{6}\right)^{j-1} \frac{1}{4^{3 j-2}} \cdot \phi_{p}^{-1}\left(\frac{1}{4^{n_{1}-1}}\right), j=1, \ldots, m_{1}-1,\right. \\
\left.\frac{1}{4^{m_{2}-1}} \cdot \phi_{q}^{-1}\left(\left(\frac{11}{6}\right)^{j-1} \frac{1}{4^{3 j-2}}\right), j=1, \ldots, n_{2}-1\right\} .
\end{array}
$$

\section{Existence of Multiple Positive Solutions}

In this section, we establish the existence of at least three positive solutions for the system of BVP (2), by using Avery's generalization of the Leggett-Williams fixed point theorem.

Let $B$ be a real Banach space with cone $P$. We define the nonnegative continuous convex functionals $\gamma, \beta$, and $\theta$ and nonnegative continuous concave functionals $\alpha$, $\psi$ on $P$, for nonnegative numbers $a^{\prime}, b^{\prime}, c^{\prime}, d^{\prime}$, and $h^{\prime}$; we define the following sets:

$$
\begin{gathered}
P\left(\gamma, c^{\prime}\right)=\left\{y \in P \mid \gamma(y)<c^{\prime}\right\}, \\
P\left(\gamma, \alpha, a^{\prime}, c^{\prime}\right)=\left\{y \in P \mid a^{\prime} \leq \alpha(y), \gamma(y) \leq c^{\prime}\right\}, \\
Q\left(\gamma, \beta, d^{\prime}, c^{\prime}\right)=\left\{y \in P \mid \beta(y) \leq d^{\prime}, \gamma(y) \leq c^{\prime}\right\}, \\
P\left(\gamma, \theta, \alpha, a^{\prime}, b^{\prime}, c^{\prime}\right) \\
=\left\{y \in P \mid a^{\prime} \leq \alpha(y), \theta(y) \leq b^{\prime}, \gamma(y) \leq c^{\prime}\right\}, \\
Q\left(\gamma, \beta, \psi, h^{\prime}, d^{\prime}, c^{\prime}\right) \\
=\left\{y \in P \mid h^{\prime} \leq \psi(y), \beta(y) \leq d^{\prime}, \gamma(y) \leq c^{\prime}\right\} .
\end{gathered}
$$

In obtaining multiple positive solutions of the BVP (2), the following so-called five-functionals fixed point theorem will be fundamental.
Theorem 10 (see [4]). Let $P$ be a cone in a real Banach space B. Suppose $\alpha$ and $\psi$ are nonnegative continuous concave functionals on $P$ and $\gamma ; \beta$ and $\theta$ are nonnegative continuous convex functionals on $P$ such that, for some positive numbers $c^{\prime}$ and $k$,

$$
\alpha(y) \leq \beta(y), \quad\|y\| \leq k \gamma(y) \quad \forall y \in \overline{P\left(\gamma, c^{\prime}\right)} .
$$

Suppose further that $T: \overline{P\left(\gamma, c^{\prime}\right)} \rightarrow \overline{P\left(\gamma, c^{\prime}\right)}$ is completely continuous, and there exist constants $h^{\prime}, d^{\prime}, a^{\prime}, b^{\prime} \geq 0$ with $0<d^{\prime}<a^{\prime}$ such that each of the following is satisfied

(B1) $\left\{y \in P\left(\gamma, \theta, \alpha, a^{\prime}, b^{\prime}, c^{\prime}\right) \mid \alpha(y)>a^{\prime}\right\} \neq \emptyset$ and $\alpha(T y)>a^{\prime}$ for $y \in P\left(\gamma, \theta, \alpha, a^{\prime}, b^{\prime}, c^{\prime}\right)$,

(B2) $\left\{y \in Q\left(\gamma, \beta, \psi, h^{\prime}, d^{\prime}, c^{\prime}\right) \mid \beta(y)<d^{\prime}\right\} \neq \emptyset$ and $\beta(T y)<d^{\prime}$ for $y \in Q\left(\gamma, \beta, \psi, h^{\prime}, d^{\prime}, c^{\prime}\right)$,

(B3) $\alpha(T y)>a^{\prime}$ provided $y \in P\left(\gamma, \alpha, a^{\prime}, c^{\prime}\right)$ with $\theta(T y)>$ $b^{\prime}$,

(B4) $\beta(T y)<d^{\prime}$ provided $y \in Q\left(\gamma, \beta, d^{\prime}, c^{\prime}\right)$ with $\psi(T y)<$ $h^{\prime}$.

Then $T$ has at least three fixed points $y_{1}, y_{2}, y_{3} \in \overline{P\left(\gamma, c^{\prime}\right)}$ such that

$$
\beta\left(y_{1}\right)<d^{\prime}, \quad a<\alpha\left(y_{2}\right), \quad d^{\prime}<\beta\left(y_{3}\right) \quad \text { with } \alpha\left(y_{3}\right)<a^{\prime} .
$$

Let $E=\{y \mid y \in C[0,1]\}$, and denote $B=E \times E$ by the norm defined as $\|(u, v)\|=\|u\|_{0}+\|v\|_{0}$, where

$$
\|y\|_{0}=\max _{t \in[0,1]}|y(t)|
$$

Then the set $B$ with the norm $\|\cdot\|$ is a complete normed linear space.

Let $I=[1 / 4,3 / 4], I_{1}=[1 / 3,2 / 3]$, and $I_{2}=[1 / 8,7 / 8]$. Define the cone $P \subset B$ by

$$
\begin{gathered}
P=\{(u, v) \in B \mid u(t) \geq 0, v(t) \geq 0, \\
\left.\min _{t \in I}(u(t)+v(t)) \geq \mathscr{M}\|(u, v)\|\right\} .
\end{gathered}
$$

Now, define the nonnegative continuous concave functionals $\alpha, \psi$ and the nonnegative continuous convex functionals $\beta, \theta$, and $\gamma$ on $P$ by

$$
\begin{aligned}
& \gamma(u, v)=\max _{t \in I_{2}}(u(t)+v(t)), \\
& \psi(u, v)=\min _{t \in I_{1}}(u(t)+v(t)), \\
& \beta(u, v)=\max _{t \in I_{1}}(u(t)+v(t)), \\
& \alpha(u, v)=\min _{t \in I}(u(t)+v(t)), \\
& \theta(u, v)=\max _{t \in I}(u(t)+v(t)) .
\end{aligned}
$$


We observe that, for any $(u, v) \in P$,

$$
\begin{aligned}
\alpha(u, v) & =\min _{t \in I}(u(t)+v(t)) \\
& \leq \max _{t \in I_{1}}(u(t)+v(t))=\beta(u, v), \\
\|(u, v)\| & =\frac{1}{\mathscr{M}} \min _{t \in I}(u(t)+v(t)) \\
& \leq \frac{1}{\mathscr{M}} \max _{t \in I_{2}}(u(t)+v(t))=\frac{1}{\mathscr{M}} \gamma(u, v) .
\end{aligned}
$$

We derive growth conditions on $f_{1}, f_{2}$ so that the BVP (2) has at least three positive solutions. We are now ready to present the main result of this section.

We denote

$$
\mathcal{S}=\int_{0}^{1} G(\tau, \tau) d \tau ; \quad \mathscr{R}=\int_{0}^{1} H(s, s) d s .
$$

Theorem 11. Suppose there exists $0<a^{\prime}<b^{\prime}<b^{\prime} / \mathscr{M} \leq c^{\prime}$ such that $f_{1}$ and $f_{2}$ satisfy the following conditions:

(A1) $\left|f_{1}(t, u, v)\right|<\phi_{p}\left(6^{m_{1}} a^{\prime} / 2 \delta\right)$ and $\left|f_{2}(t, u, v)\right|<6^{n_{2}} \phi_{q}$ $\left(a^{\prime} / 2 \mathscr{R}\right)$, for all $t \in[0,1]$ and $u, v \in\left[\mathscr{M} a^{\prime}, a^{\prime}\right]$,

(A2) $\left|f_{1}(t, u, v)\right|>4^{n_{1}-1} \phi_{p}\left(b^{\prime} 6^{m_{1}} 4^{3 m_{1}-2} / \delta 11^{m_{1}-1}\right)$ for all $t \in[1 / 4,3 / 4]$ and $u, v \in\left[b^{\prime}, b^{\prime} / \mathscr{M}\right]$ or

$\left|f_{2}(t, u, v)\right|>\left(6^{n_{2}} 4^{3 n_{2}-2} / 11^{n_{2}-1}\right) \phi_{q}\left(b^{\prime} 4^{m_{2}} / \mathscr{R}\right)$ for all $t \in[1 / 4,3 / 4]$ and $u, v \in\left[b^{\prime}, b^{\prime} / \mathscr{M}\right]$,

(A3) $\left|f_{1}(t, u, v)\right|<\phi_{p}\left(6^{m_{1}} c^{\prime} / 2 \mathcal{S}\right)$ and $\left|f_{2}(t, u, v)\right|<6^{n_{2}} \phi_{q}$ $\left(c^{\prime} / 2 \mathscr{R}\right)$, for all $t \in[0,1]$ and $u, v \in\left[0, c^{\prime}\right]$.

Then the BVP (2) has at least three positive solutions.

Proof. Define the completely continuous operator $T: P \rightarrow$ $B$ by

$$
\begin{aligned}
& T(u, v) \\
& :=\left(\int_{0}^{1} H_{m_{1}}(t, s) \phi_{q}\left(\int_{0}^{1} G(s, \tau) f_{1}(\tau, u(\tau), v(\tau)) d \tau\right) d s,\right. \\
& \left.\quad \int_{0}^{1} H(t, s) \phi_{p}\left(\int_{0}^{1} G_{n_{2}}(s, \tau) f_{2}(\tau, u(\tau), v(\tau)) d \tau\right) d s\right) .
\end{aligned}
$$

And also we denote

$$
\begin{aligned}
& T_{1}(u, v):= \int_{0}^{1} H_{m_{1}}(t, s) \phi_{q} \\
& \times\left(\int_{0}^{1} G(s, \tau) f_{1}(\tau, u(\tau), v(\tau)) d \tau\right) d s \\
& T_{2}(u, v):=\int_{0}^{1} H(t, s) \phi_{p} \\
& \quad \times\left(\int_{0}^{1} G_{n_{2}}(s, \tau) f_{2}(\tau, u(\tau), v(\tau)) d \tau\right) d s .
\end{aligned}
$$

It is obvious that a fixed point of $T$ is a solution of the BVP (2). We seek three fixed points $\left(x_{1}, x_{2}\right),\left(y_{1}, y_{2}\right)$, and $\left(z_{1}, z_{2}\right) \in$ $P$ of $T$. First, we show that $T: P \rightarrow P$. Let $(u, v) \in P$. Clearly, $T_{1}(u, v)(t) \geq 0, T_{2}(u, v)(t) \geq 0$ for $t \in[0,1]$. Consider

$$
\begin{aligned}
\min _{t \in I} T(u, v) & =\min _{t \in I}\left(T_{1}(u, v)(t)+T_{2}(u, v)(t)\right) \\
& =\min _{t \in I} T_{1}(u, v)(t)+\min _{t \in I} T_{2}(u, v)(t) \\
& \geq \min _{t \in I} \mathscr{M}\|T(u, v)\|_{0}+\min _{t \in I} \mathscr{M}\|T(u, v)\|_{0} \\
& =\mathscr{M}\|T(u, v)\| .
\end{aligned}
$$

Thus, $T: P \rightarrow P$. Next, for all $(u, v) \in P$, by (46), (47), respectively, we have $\alpha(u, v) \leq \beta(u, v)$ and $\|(u, v)\| \leq$ $(1 / \mathscr{M}) \gamma(u, v)$. To show that $T: \overline{P\left(\gamma, c^{\prime}\right)} \rightarrow \overline{P\left(\gamma, c^{\prime}\right)}$, let $(u, v) \in \overline{P\left(\gamma, c^{\prime}\right)}$. This implies $\|(u, v)\| \leq(1 / \mathscr{M}) c^{\prime}$. We may now use condition (A3) to obtain

$$
\begin{aligned}
& \gamma\left(T_{1}(u, v), T_{2}(u, v)\right) \\
& =\max _{t \in I_{2}}\left(T_{1}(u, v)(t)+T_{2}(u, v)(t)\right) \\
& =\max _{t \in I_{2}} \int_{0}^{1} H_{m_{1}}(t, s) \phi_{p}^{-1} \\
& \times\left(\int_{0}^{1} G(s, \tau) f_{1}(\tau, u(\tau), v(\tau)) d \tau\right) d s \\
& +\max _{t \in I_{2}} \int_{0}^{1} H(t, s) \phi_{q}^{-1} \\
& \times\left(\int_{0}^{1} G_{n_{2}}(s, \tau) f_{2}(\tau, u(\tau), v(\tau)) d \tau\right) d s \\
& <\max _{t \in I_{2}} \frac{1}{6^{m_{1}-1}} \int_{0}^{1} s(1-s) \phi_{p}^{-1} \\
& \times\left(\int_{0}^{1} G(\tau, \tau) f_{1}(\tau, u(\tau), v(\tau)) d \tau\right) d s \\
& +\max _{t \in I_{2}} \int_{0}^{1} H(s, s) \phi_{q}^{-1} \\
& \times\left(\int_{0}^{1} \frac{1}{6^{n_{2}-1}} \tau(1-\tau) f_{2}(\tau, u(\tau), v(\tau)) d \tau\right) d s \\
& =\frac{1}{6^{m_{1}-1}} \phi_{p}^{-1}\left(\int_{0}^{1} G(\tau, \tau) f_{1}(\tau, u(\tau), v(\tau)) d \tau\right) \\
& \times \int_{0}^{1} s(1-s) d s \\
& +\int_{0}^{1} H(s, s) \phi_{q}^{-1} \\
& \times\left(\frac{1}{6^{n_{2}-1}} \int_{0}^{1} \tau(1-\tau) f_{2}(\tau, u(\tau), v(\tau)) d \tau\right) d s
\end{aligned}
$$




$$
\begin{aligned}
& =\frac{1}{6^{m_{1}}} \phi_{p}^{-1}\left(\int_{0}^{1} G(\tau, \tau) f_{1}(\tau, u(\tau), v(\tau)) d \tau\right) \\
& +\int_{0}^{1} H(s, s) \phi_{q}^{-1} \\
& \quad \times\left(\frac{1}{6^{n_{2}-1}} \int_{0}^{1} \tau(1-\tau) f_{2}(\tau, u(\tau), v(\tau)) d \tau\right) d s \leq c^{\prime} .
\end{aligned}
$$

Therefore, $T: \overline{P\left(\gamma, c^{\prime}\right)} \rightarrow \overline{P\left(\gamma, c^{\prime}\right)}$.

We first verify that condition (B1) of Theorem 10 is satisfied. The constant function is

$$
\begin{aligned}
& \frac{b^{\prime}+b^{\prime} / \mathscr{M}}{2} \\
& \quad \in\left\{(u, v) \in P\left(\gamma, \theta, \alpha, b^{\prime}, \frac{b^{\prime}}{\mathscr{M}}, c^{\prime}\right) \mid \alpha(u, v)>b^{\prime}\right\} \neq \emptyset .
\end{aligned}
$$

Next, let $(u, v) \in P\left(\gamma, \theta, \alpha, b^{\prime}, b^{\prime} / \mathscr{M}, c^{\prime}\right)$. It follows that from (A2)

$$
\begin{aligned}
& \alpha\left(T_{1}(u, v), T_{2}(u, v)\right) \\
& =\min _{t \in I}\left(T_{1}(u, v)(t)+T_{2}(u, v)(t)\right) \\
& \geq \min _{t \in I} T_{1}(u, v)(t) \\
& =\min _{t \in I} \int_{0}^{1} H_{m_{1}}(t, s) \phi_{p}^{-1} \\
& \times\left(\int_{0}^{1} G(s, \tau) f_{1}(\tau, u(\tau), v(\tau)) d \tau\right) d s \\
& \geq \int_{0}^{1}\left(\frac{11^{m_{1}-1} s(1-s)}{6^{m_{1}-1} 4^{3 m_{1}-2}}\right) \phi_{p}^{-1} \\
& \times\left(\int_{0}^{1} \frac{1}{4^{n_{1}-1}} G(\tau, \tau) f_{1}(\tau, u(\tau), v(\tau)) d \tau\right) d s \\
& =\left(\frac{11^{m_{1}-1}}{6^{m_{1}-1} 4^{3 m_{1}-2}}\right) \phi_{p}^{-1} \\
& \times\left(\int_{0}^{1} \frac{1}{4^{n_{1}-1}} G(\tau, \tau) f_{1}(\tau, u(\tau), v(\tau)) d \tau\right) \\
& \times \int_{0}^{1} s(1-s) d s \\
& \geq\left(\frac{11^{m_{1}-1}}{6^{m_{1}} 4^{3 m_{1}-2}}\right) \phi_{p}^{-1}\left(\frac{1}{4^{n_{1}-1}}\right) \phi_{p}^{-1} \\
& \times\left(\int_{0}^{1} G(\tau, \tau) f_{1}(\tau, u(\tau), v(\tau)) d \tau\right)=b^{\prime} .
\end{aligned}
$$

Similarly,

$$
\begin{aligned}
& \min _{t \in I} T_{2}(u, v)(t) \\
&=\min _{t \in I} \int_{0}^{1} H(t, s) \phi_{q}^{-1} \\
& \quad \times\left(\int_{0}^{1} G_{n_{2}}(s, \tau) f_{2}(\tau, u(\tau), v(\tau)) d \tau\right) d s \\
& \geq \int_{0}^{1} \frac{1}{4^{m_{2}-1}} H(s, s) \phi_{q}^{-1} \\
& \quad \times\left(\int_{0}^{1} \frac{11^{n_{2}-1} \tau(1-\tau)}{6^{n_{2}-1} 4^{3 n_{2}-2}} f_{2}(\tau, u(\tau), v(\tau)) d \tau\right) d s \\
& \geq \frac{1}{4^{m_{2}-1}} \phi_{q}^{-1} \\
& \quad \times\left(\frac{11^{n_{2}-1}}{6^{n_{2}-1} 4^{3 n_{2}-2}} \int_{0}^{1} \tau(1-\tau) f_{2}(\tau, u(\tau), v(\tau)) d \tau\right) \\
& \quad \times \int_{0}^{1} H(s, s) d s=b^{\prime},
\end{aligned}
$$

and, hence, we have $\alpha\left(T_{1}(u, v), T_{2}(u, v)\right) \geq b^{\prime}$.

Next, we show that (B2) is fulfilled. The constant function is

$$
\begin{aligned}
& \frac{a^{\prime} \mathscr{M}+a^{\prime}}{2} \\
& \quad \in\left\{(u, v) \in Q\left(\gamma, \beta, \psi, a^{\prime} \mathscr{M}, a^{\prime}, c^{\prime}\right) \mid \beta\left(v_{1}, v_{2}\right)<a^{\prime}\right\} \neq \emptyset .
\end{aligned}
$$

Let $(u, v) \in Q\left(\gamma, \beta, \psi, a^{\prime} \mathscr{M}, a^{\prime}, c^{\prime}\right)$. And from (A1),

$$
\begin{aligned}
& \beta\left(T_{1}(u, v), T_{2}(u, v)\right) \\
& =\max _{t \in I_{1}}\left(T_{1}(u, v)(t)+T_{2}(u, v)(t)\right) \\
& =\max _{t \in I_{1}} \int_{0}^{1} H_{m}(t, s) \phi_{p}^{-1} \\
& \quad \times\left(\int_{0}^{1} G(s, \tau) f_{1}(\tau, u(\tau), v(\tau)) d \tau\right) d s \\
& \quad+\max _{t \in I_{1}} \int_{0}^{1} H(t, s) \phi_{q}^{-1} \\
& \quad \times\left(\int_{0}^{1} G_{n}(s, \tau) f_{2}(\tau, u(\tau), v(\tau)) d \tau\right) d s \\
& \leq \max _{t \in I_{1}} \frac{1}{6^{m_{1}-1}} \int_{0}^{1} s(1-s) \phi_{p}^{-1} \\
& \times\left(\int_{0}^{1} G(\tau, \tau) f_{1}(\tau, u(\tau), v(\tau)) d \tau\right) d s
\end{aligned}
$$




$$
\begin{aligned}
& +\max _{t \in I_{1}} \int_{0}^{1} H(s, s) \phi_{q}^{-1} \\
& \quad \times\left(\int_{0}^{1} \frac{1}{6^{n_{2}-1}} \tau(1-\tau) f_{2}(\tau, u(\tau), v(\tau)) d \tau\right) d s \\
& =\frac{1}{6^{m_{1}-1}} \phi_{p}^{-1}\left(\int_{0}^{1} G(\tau, \tau) f_{1}(\tau, u(\tau), v(\tau)) d \tau\right) \\
& \quad \times \int_{0}^{1} s(1-s) d s \\
& +\int_{0}^{1} H(s, s) \phi_{q}^{-1} \\
& \quad \times\left(\frac{1}{6^{n_{2}-1}} \int_{0}^{1} \tau(1-\tau) f_{2}(\tau, u(\tau), v(\tau)) d \tau\right) d s \\
& =\frac{1}{6^{m_{1}}} \phi_{p}^{-1}\left(\int_{0}^{1} G(\tau, \tau) f_{1}(\tau, u(\tau), v(\tau)) d \tau\right) \\
& \quad+\int_{0}^{1} H(s, s) \phi_{q}^{-1} \\
& \quad \times\left(\frac{1}{6^{n_{2}-1}} \int_{0}^{1} \tau(1-\tau) f_{2}(\tau, u(\tau), v(\tau)) d \tau\right) d s=a^{\prime} .
\end{aligned}
$$

To see that (B3) is satisfied, let $\left(v_{1}, v_{2}\right) \in P\left(\gamma, \alpha, b^{\prime}, c^{\prime}\right)$ with

$$
\theta\left(T_{1}\left(v_{1}, v_{2}\right), T_{2}\left(v_{1}, v_{2}\right)\right)>\frac{b^{\prime}}{\mathscr{M}}
$$

Using (23), (27), (31), and (35), we get

$$
\begin{aligned}
& \alpha\left(T_{1}(u, v), T_{2}(u, v)\right) \\
& =\min _{t \in I}\left(T_{1}(u, v)(t)+T_{2}(u, v)(t)\right) \\
& =\min _{t \in I} \int_{0}^{1} H_{m_{1}}(t, s) \phi_{p}^{-1} \\
& \quad \times\left(\int_{0}^{1} G(s, \tau) f_{1}(\tau, u(\tau), v(\tau)) d \tau\right) d s \\
& \quad \min _{t \in I} \int_{0}^{1} H(t, s) \phi_{q}^{-1} \\
& \quad \times\left(\int_{0}^{1} G_{n_{2}}(s, \tau) f_{2}(\tau, u(\tau), v(\tau)) d \tau\right) d s \\
& \geq \int_{0}^{1}\left(\frac{11^{m_{1}-1} s(1-s)}{6^{m_{1}-1} 4^{3 m_{1}-2}}\right) \phi_{p}^{-1} \\
& \quad \times\left(\int_{0}^{1} \frac{1}{4^{n_{1}-1}} G(\tau, \tau) f_{1}(\tau, u(\tau), v(\tau)) d \tau\right) d s \\
& \quad+\int_{0}^{1} \frac{1}{4^{m_{2}-1}} H(s, s) \phi_{q}^{-1}
\end{aligned}
$$

$$
\begin{aligned}
& \times\left(\int_{0}^{1} \frac{11^{n_{2}-1} \tau(1-\tau)}{6^{n_{2}-1} 4^{3 n_{2}-2}} f_{2}(\tau, u(\tau), v(\tau)) d \tau\right) d s \\
= & \left(\frac{11^{m_{1}-1}}{6^{m_{1}-1} 4^{3 m_{1}-2}}\right) \phi_{p}^{-1} \\
& \times\left(\int_{0}^{1} \frac{1}{4^{n_{1}-1}} G(\tau, \tau) f_{1}(\tau, u(\tau), v(\tau)) d \tau\right) \\
& \times \int_{0}^{1} s(1-s) d s \\
& +\frac{1}{4^{m_{2}-1}} \phi_{q}^{-1} \\
& \times\left(\frac{11^{n_{2}-1}}{6^{n_{2}-1} 4^{3 n_{2}-2}} \int_{0}^{1} \tau(1-\tau) f_{2}(\tau, u(\tau), v(\tau)) d \tau\right) \\
& \times \int_{0}^{1} H(s, s) d s \\
= & \mathscr{M} \theta\left(T_{1}(u, v), T_{2}(u, v)\right)>b^{\prime} .
\end{aligned}
$$

Finally, we show that (B4) holds. Let $(u, v) \in Q\left(\gamma, \beta, a^{\prime}, c^{\prime}\right)$ with $\psi\left(T_{1}(u, v), T_{2}(u, v)\right)<a^{\prime} / \mathscr{M}$. Using (23), (27), (31), and (35), we have

$$
\begin{aligned}
& \beta\left(T_{1}(u, v), T_{2}(u, v)\right) \\
& =\max _{t \in I_{1}}\left(T_{1}(u, v)(t)+T_{2}(u, v)(t)\right) \\
& =\max _{t \in I_{1}} \int_{0}^{1} H_{m_{1}}(t, s) \phi_{p}^{-1} \\
& \quad \times\left(\int_{0}^{1} G(s, \tau) f_{1}(\tau, u(\tau), v(\tau)) d \tau\right) d s \\
& +\max _{t \in I_{1}} \int_{0}^{1} H(t, s) \phi_{q}^{-1} \\
& \quad \times\left(\int_{0}^{1} G_{n_{2}}(s, \tau) f_{2}(\tau, u(\tau), v(\tau)) d \tau\right) d s \\
& \quad \times\left(\int_{0}^{1} \frac{11^{m_{1}-1} s(1-s)}{6^{m_{1}-1} 4^{3 m_{1}-2}}\right) \phi_{p}^{-1} \\
& \left.+\int_{0}^{1} \frac{1}{4^{m_{1}-1}-1} H(\tau, \tau) f_{1}(\tau, u(\tau), v(\tau)) d \tau\right) d s \\
& \quad \times\left(\int_{0}^{1} \frac{11^{n_{2}-1} \tau(1-\tau)}{6^{n_{2}-1} 4^{3 n_{2}-2}} f_{2}(\tau, u(\tau), v(\tau)) d \tau\right) d s
\end{aligned}
$$




$$
\begin{aligned}
& =\left(\frac{11^{m_{1}-1}}{6^{m_{1}-1} 4^{3 m_{1}-2}}\right) \phi_{p}^{-1} \\
& \quad \times\left(\int_{0}^{1} \frac{1}{4^{n_{1}-1}} G(\tau, \tau) f_{1}(\tau, u(\tau), v(\tau)) d \tau\right) \\
& \quad \times \int_{0}^{1} s(1-s) d s \\
& \quad+\frac{1}{4^{m_{2}-1}} \phi_{q}^{-1} \\
& \quad \times\left(\frac{11^{n_{2}-1}}{6^{n_{2}-1} 4^{3 n_{2}-2}} \int_{0}^{1} \tau(1-\tau) f_{2}(\tau, u(\tau), v(\tau)) d \tau\right) \\
& \quad \times \int_{0}^{1} H(s, s) d s \\
& =\mathscr{M} \psi\left(T_{1}(u, v), T_{2}(u, v)\right)>a^{\prime} .
\end{aligned}
$$

We have proved that all the conditions of Theorem 10 are satisfied, and so there exist at least three positive solutions. Therefore the BVP (2) has at least three positive solutions. This completed the proof of the theorem.

\section{Example}

Considering the higher order $(p, q)$-Laplacian two-point boundary value problem,

$$
\begin{gathered}
(-1)^{4}\left[\phi_{p}\left(u^{\prime \prime}(t)\right)\right]^{\prime \prime \prime}=f_{1}(t, u(t), v(t)), \\
t \in[0,1], \\
(-1)^{2}\left[\phi_{q}\left(v^{\prime}(t)\right)\right]^{\prime \prime}=f_{2}(t, u(t), v(t)), \frac{1}{p}+\frac{1}{q}=1, \\
u(0)=0=u(1), \\
{\left[\phi_{p}\left(u^{\prime \prime}(t)\right)\right]_{\mathrm{at} t=0}^{(j)}=0, \quad j=0,1,} \\
{\left[\phi_{p}\left(u^{\prime \prime}(t)\right)\right]_{\mathrm{at} t=1}=0,} \\
{\left[\phi_{q}\left(v^{\prime}(t)\right)\right]_{\mathrm{at} t=0}=0=\left[\phi_{q}\left(v^{\prime}(t)\right)\right]_{\mathrm{at} t=1},} \\
v(1)=0,
\end{gathered}
$$

where

$$
\begin{aligned}
f_{1}(t, u(t), v(t)) \\
\quad= \begin{cases}12,098.134 u v^{2}-577.134, & u, v \in[0.015625,1], \\
0.267 u+11,520.73, & u, v \in[1,16] \\
283.56 u+16,061.89, & u, v \in[16,43]\end{cases}
\end{aligned}
$$

$$
\begin{aligned}
f_{2}(t, u(t), v(t)) \\
= \begin{cases}21.34 u^{2} v+3.67, & u, v \in[0.015625,1] \\
0.34 v+24.67, & u, v \in[1,16] \\
170.85 v-2,703.63, & u, v \in[16,43]\end{cases}
\end{aligned}
$$

are continuous functions from $[0,1] \times \mathbb{R}^{2}$ to $\mathbb{R}$. A simple calculation shows that $\mathscr{M}=0.0625,(p=2), \mathcal{S}=1 / 30$, and $\mathscr{R}=1$. If we choose $a^{\prime}=1 / 4, b^{\prime}=1$, and $c^{\prime}=43$, then conditions (A1)-(A3) are satisfied. Therefore, it follows from Theorem 11 that the BVP (61) has at least three positive solutions.

\section{Acknowledgment}

One of the authors (Dr. Penugurthi Murali) is thankful to CSIR, India, for awarding him a Research Associate.

\section{References}

[1] R. P. Agarwal, D. O’Regan, and P. J. Y. Wong, Positive Solutions of Differential, Difference and Integral Equations, Kluwer Academic Publishers, Dordrecht, The Netherlands, 1999.

[2] P. V. S. Anand, P. Murali, and K. R. Prasad, "Multiple symmetric positive solutions for systems of higher order boundary-value problems on time scales," Electronic Journal of Differential Equations, vol. 2011, no. 102, pp. 1-12, 2011.

[3] D. R. Anderson, "Eigenvalue intervals for even-order SturmLiouville dynamic equations," Communications on Applied Nonlinear Analysis, vol. 12, no. 4, pp. 1-13, 2005.

[4] R. I. Avery, "A generalization of the Leggett-Williams fixed point theorem," Mathematical Sciences Research Hot-Line, vol. 3, no. 7, pp. 9-14, 1999.

[5] R. I. Avery, J. M. Davis, and J. Henderson, "Three symmetric positive solutions for Lidstone problems by a generalization of the Leggett-Williams theorem," Electronic Journal of Differential Equations, vol. 2000, no. 40, pp. 1-15, 2000.

[6] J. M. Davis, J. Henderson, and P. J. Y. Wong, "General Lidstone problems: multiplicity and symmetry of solutions," Journal of Mathematical Analysis and Applications, vol. 251, no. 2, pp. 527$548,2000$.

[7] L. H. Erbe and H. Wang, "On the existence of positive solutions of ordinary differential equations," Proceedings of the American Mathematical Society, vol. 120, no. 3, pp. 743-748, 1994.

[8] D. J. Guo and V. Lakshmikantham, Nonlinear Problems in Abstract Cones, vol. 5 of Notes and Reports in Mathematics in Science and Engineering, Academic Press, Boston, Mass, USA, 1988.

[9] J. Henderson, P. Murali, and K. R. Prasad, "Multiple symmetric positive solutions for two-point even order boundary value problems on time scales," Mathematics in Engineering, Science and Aerospace, vol. 1, no. 1, pp. 105-117, 2010.

[10] J. Henderson and H. B. Thompson, "Multiple symmetric positive solutions for a second order boundary value problem," Proceedings of the American Mathematical Society, vol. 128, no. 8, pp. 2373-2379, 2000

[11] K. R. Prasad, P. Murali, and N. V. V. S. Suryanarayana, "Multiple positive solutions for the system of higher order two-point boundary value problems on time scales," Electronic Journal of 
Qualitative Theory of Differential Equations, vol. 2009, no. 32, pp. 1-13, 2009.

[12] K. R. Prasad and P. Murali, "Solvability of $p$-Laplacian singular boundary value problems on time scales," Advances in Pure and Applied Mathematics, vol. 3, no. 4, pp. 377-391, 2012. 


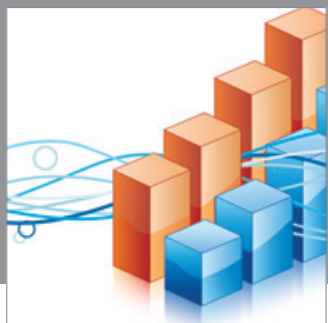

Advances in

Operations Research

mansans

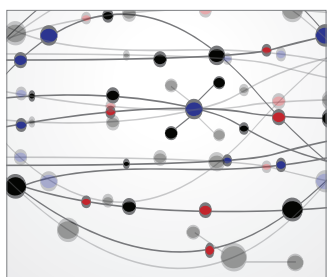

The Scientific World Journal
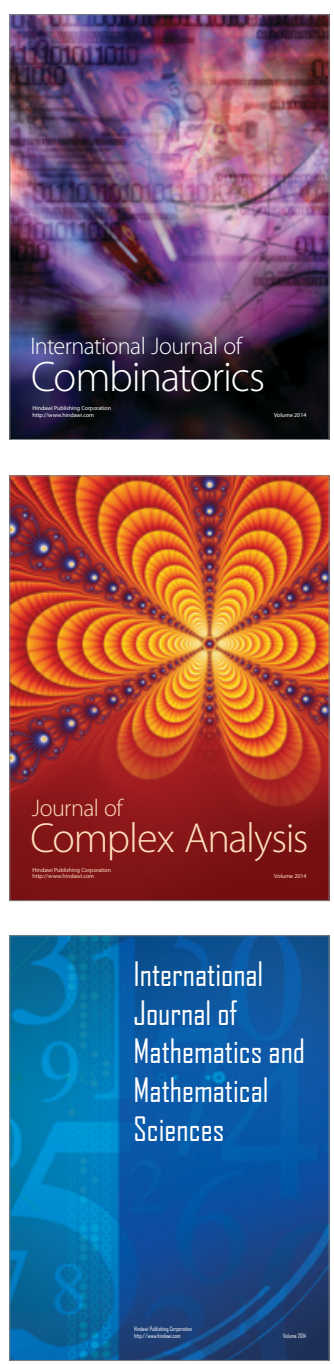
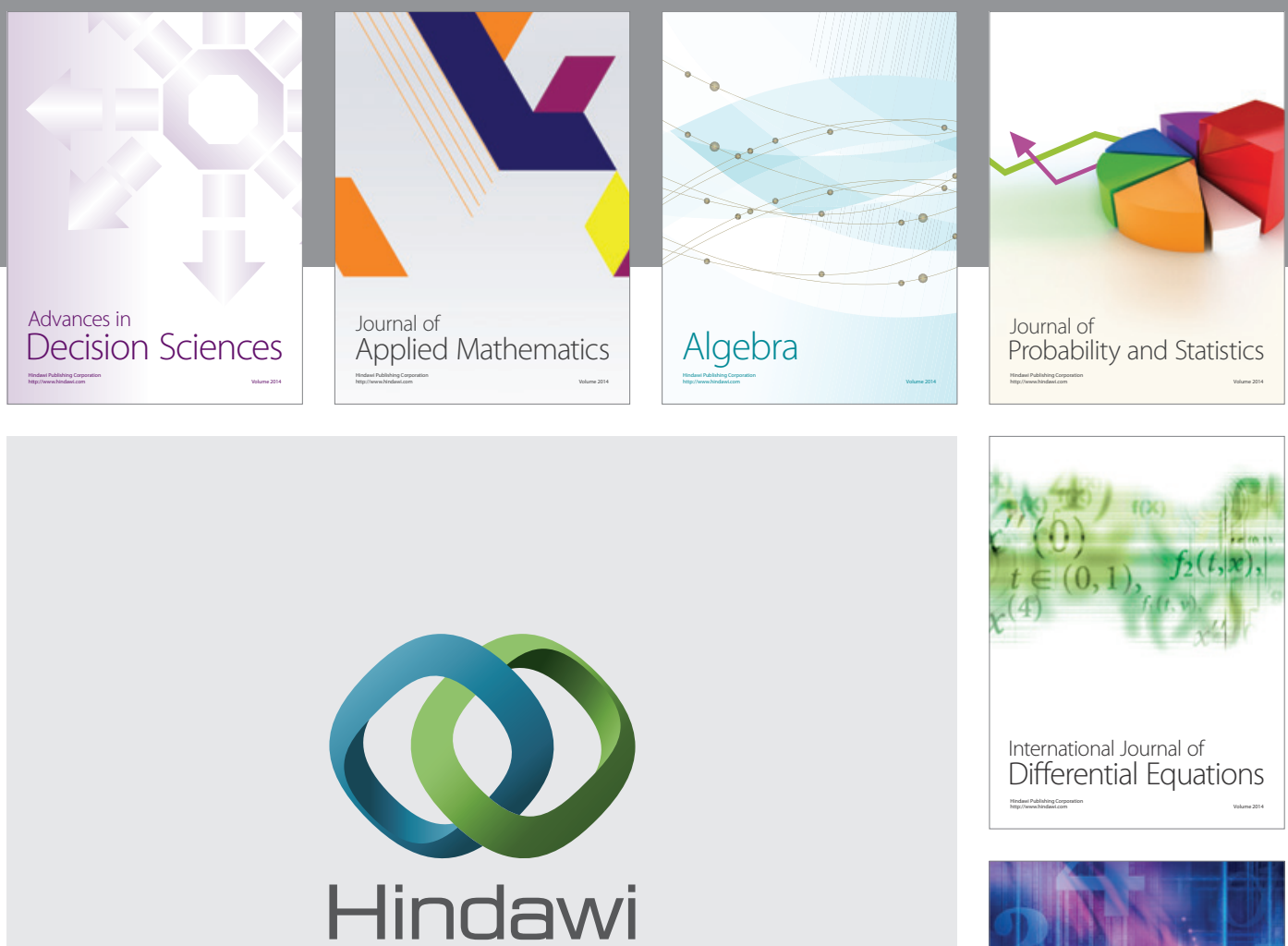

Submit your manuscripts at http://www.hindawi.com
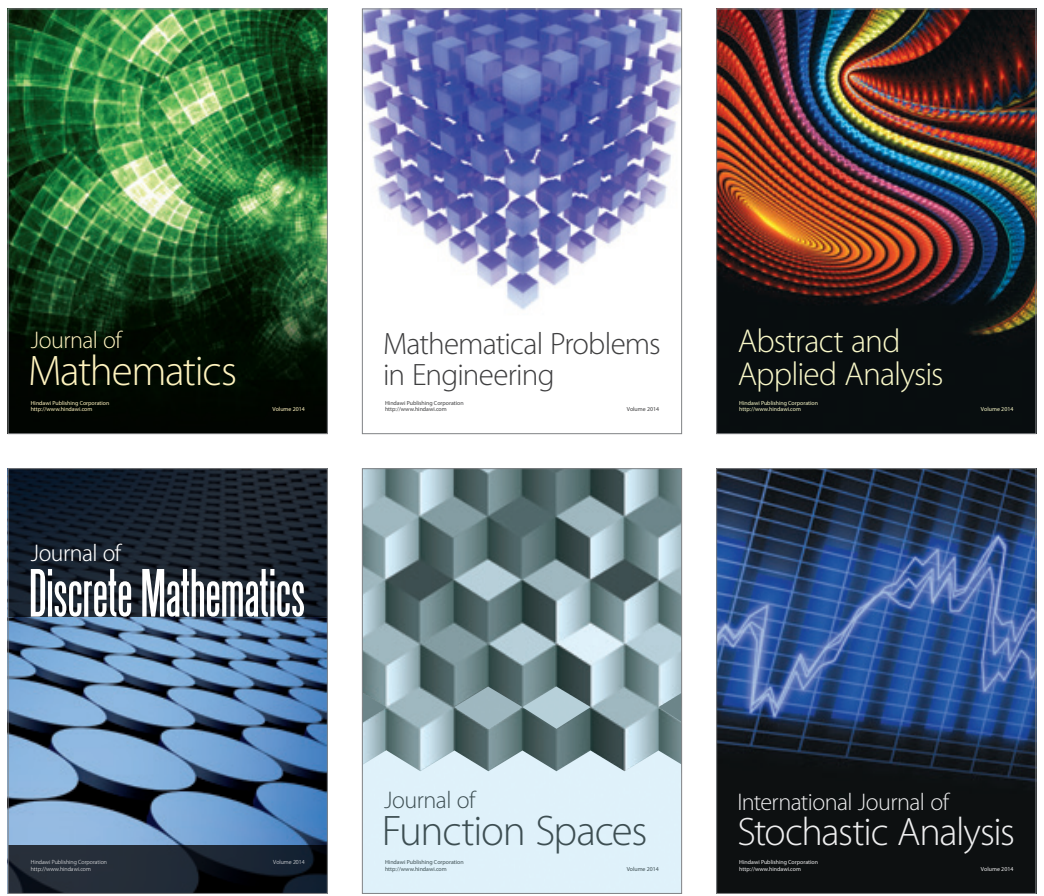

Journal of

Function Spaces

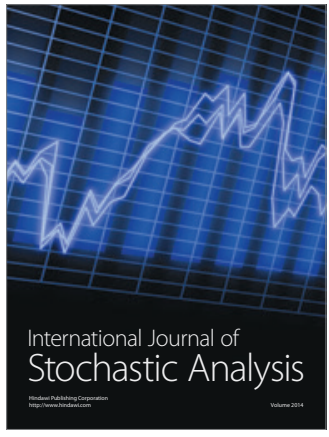

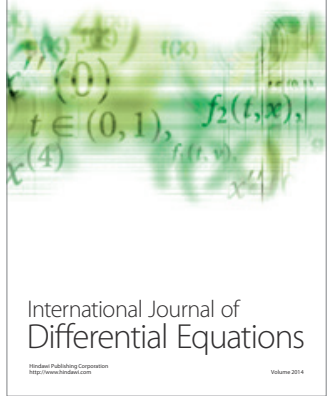
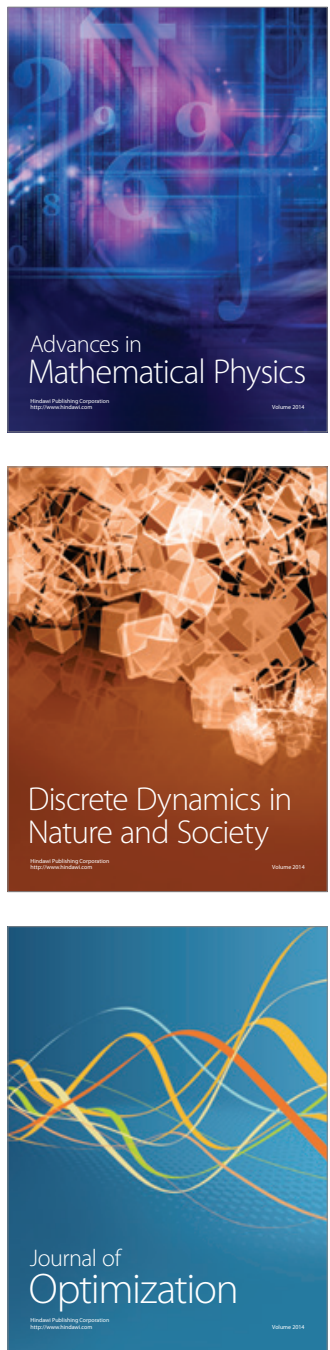